Relations industrielles

Industrial Relations

\title{
Le message de la responsabilité sociale
}

\section{Vittorio Vaccari}

Volume 5, numéro 3, décembre 1949

URI : https://id.erudit.org/iderudit/1023293ar

DOI : https://doi.org/10.7202/1023293ar

Aller au sommaire du numéro

Éditeur(s)

Département des relations industrielles de l'Université Laval

ISSN

0034-379X (imprimé)

1703-8138 (numérique)

Découvrir la revue

Citer cet article

Vaccari, V. (1949). Le message de la responsabilité sociale. Relations

industrielles / Industrial Relations, 5(3), 22-24. https://doi.org/10.7202/1023293ar

Tous droits réservés (C Département des relations industrielles de l’Université Laval, 1949
Ce document est protégé par la loi sur le droit d'auteur. L'utilisation des services d'Érudit (y compris la reproduction) est assujettie à sa politique d'utilisation que vous pouvez consulter en ligne.

https://apropos.erudit.org/fr/usagers/politique-dutilisation/ 
La véritable signification

d'un message de Pie XII aux patrons catholiques

\title{
LE MESSAGE de LA RESPONSABILITÉ SOCIALE
}

\author{
VitTorio Vaccari
}

On a dit déjà que le discours du Pape Pie XII aux patrons chrétiens ${ }^{1}$ est un grand document social. Il doit être interprêté dans le cadre des directives sociales que, depuis l'encyclique Rerum Novarum à nos jours, la papauté n'a jamais cessé de donner aux peuples de la terre, afin que la technique ne trahisse pas la conscience, et que le pouvoir économique ne vienne pas suffoquer les exigences humaines des forces personnelles qui vivent dans le monde économique.

Il y a quelqu'un qui a voulu injustement relever dans ce discours un changement d'orientation en regard des principes énoncés dans d'autres documents, et surtout dans les messages de Noël radiodiffusés durant les années de la guerre.

Il y en a encore, dans divers pays, qui ont voulu délibérément ignorer ce discours et ces affirmations des principes, surtout en ce qui a trait à l'ordre, à la structure juridique de l'entreprise et à son caractère privé. Et il faut dire qu'il $y$ a eu certains milieux de patrons qui, se bornant à un examen superficiel et fragmentaire du discours, ont mutilé sa construction organique, de telle façon qu'ils l'ont interprété comme un exposition des droits naturels du patron plutôt que de ses devoirs également.

Comme nous l'avons dit, on ne peut interpréter ce discours autrement que dans le cadre de toute la doctrine sociale pontificale, exprimée dans les documents qui l'ont précédé et, aussi, dans ceux tout récents qui l'ont suivi, en particulier dans le radio-message à l'Allemagne catholique du 4 septembre et l'allocution adressée une semaine après aux pèlerins du Mouvement ouvrier belge.

Dans ce dernier discours, le Saint-Père a voulu faire ressortir que le décret de condamnation et d'excommunication du communisme n'a rien à voir avec «l'opposition entre riche ou pauvre, entre capitaliste et prolétaire, entre propriétaires et ceux qui ne possèdent rien ». Et Il a montré comment il ne faut pas que le monde ouvrier tombe dans le communisme athée.

(1) PIE XII, Discours du 7 mai 1949, Bulletin des relations industrielles, Vol. 4, no 9, pp. 81-83.

\section{Le point central de la doctrine sociale chrétienne}

Le point central de la doctrine sociale chrétienne reste la personne humaine, avec ses aspirations d'ordre moral et avec ses exigences matérielles. Ces dernières demeurent le moyen indispensable pour le développement des premières. Personne ne peut enseigner la vertu à celui qui n'a pas le nécessaire pour vivre. Personne ne peut exiger la tranquillité de celui qui n'a pas une possibilité minime de sécurité économique et une garantie suffisante pour le travail du lendemain.

C'est pour cela qu'au cours des temps, la Papauté répète les directives tendant à fournir à la personne humaine du travailleur une existence conforme à sa dignité morale et à ses besoins économiques, et à procurer à l'ensemble des travailleurs une parité juridique et morale d'association et d'expression destinée à sauvegarder leurs droits et « à les maintenir au niveau des exigences modernes ».

Ainsi, la doctrine sociale chrétienne a affirmé le droit à une rémunération familiale, par delà la conception individualiste du salaire-prestation. Elle a affirmé le droit à une habitation saine, à une habitation convenable, à une instruction professionnelle et à la sécurité sociale, et enfin elle a exprimé la suggestion qu'on dépasse le régime du salariat pour permettre un meilleur et plus complet développement de la personnalité de l'ouvrier. C'est à ce point que souvent surgit l'équivoque sur le plan pratique. Une équivoque qui naît et se multiplie alors que des considérations unilatérales et des conjectures politiques extraordinaires influencent le monde social qui, au contraire, devrait voir les rencontres de la loi morale avec les exigences techniques de l'organisation économique et productive.

A ce moment-là la conception de la justice sociale, si souvent invoquée, devient parfois une « fausse idée claire ». Il n'est pas nécessaire de 
rappeler que souvent, selon les circonstances, cette conception de la justice sociale reste identifiée avec la revendication syndicale ou avec un absurde nivellement des richesses matérielles, ou bien avec un pur et simple renversement de l'ordre économique actuel. C'est ainsi qu'on parle de justice sociale comme si elle était l'antithèse des valeurs sociales actuelles et non pas l'orientation perfectible d'un ordre économique fondé sur les bases naturelles de la société et qui résulte des composantes des possibilités économiques productives et des exigences morales et économiques de la collectivité. Alors, la valeur intrinsèque de ces principes tend plutôt à résider dans les idées du bouleversement que dans celles d'un meilleur ordre final de la société.

Il va sans dire que ceci est une conception matérialiste qui néglige ce fait que les relations sociales s'expriment d'abord dans la réalité morale de chaque jour et qu'elles trouvent leur première racine dans la conscience de chaque individu. Il faut souligner pourtant que les conséquences d'une telle conception sont désastreuses. Les méthodes du collectivisme économique, même privées du bagage de la doctrine marxiste, sont suffisantes pour renverser dans ses bases l'ordre social.

Quand l'intransigeance des classes est exaspérée au point de rendre impossible toute collaboration entre patrons et ouvriers, quand les revendications vont au delà de toute possibilité raisonnable de l'entreprise, quand le syndicat sort de sa fonction naturelle et use de sa force pour faire une pression sur l'Etat, le péril est grave pour toute la collectivité. C'est vrai que l'organisation productive et l'entreprise se trouvent aujourd'hui en face de perspectives de rapide évolution qui permettront à la personne humaine du travailleur de prendre la place qui lui est due. Mais il est indispensable qu'une telle évolution soit une évolution vers le mieux qui ne tende pas seulement à un relèvement économique, mais encore à un progrès moral. Si l'on ne tient pas compte de cela, les réformes dites de «structure », malgré la perfection technique de leur plan, deviennent une expression dénuée de sens.

Quand on dit: changeons radicalement l'ordre actuel et nous obtiendrons un déplacement dans la distribution des valeurs actuelles, on ne dit rien du tout. En effet, le déplacement peut être négatif. Et souvent il tend à rester négatif, ou parce qu'on ignore les nécessités de l'organisation productive, ou parce qu'on oublie (comme si elles étaient inutiles) les responsabilités et les difficul- tés que rencontrent ceux qui sont appelés à diriger et à produire.

\section{Le message de la responsabilité sociale}

Quiconque connaît l'histoire la plus récente peut se rendre compte que parfois l'individualisme économique a été la cause directe des mouvements révolutionnaires, et qu'il a aidé les planifications collectivistes à causer un abaissement du niveau moyen de vie de la collectivité.

On observe une décadence générale du sens de la « responsabilité » dans le domaine économique et social. Il faut dire que cette décadence doit être attribuée en partie aux patrons. Quelques-uns d'entre eux, au moins dans les pays d'Europe, ont été de fait, consciemment ou non, les propagateurs d'une théorie et d'une pratique nettement individualistes. Ils n'ont pas compris leur rôle social, et quand ils ont pris en considération les exigences sociales des ouvriers, ils ne l'ont pas fait par un acte spontané, mais sous la pression de la force des organisations ouvrières ou par crainte de quelque parti extrémiste. Au fond, ces façons d'agir, faites d'attentes, de concessions imposées, d'égoïsme aveugle, ont créé une atmosphère tendue d'opposition dans le monde de la production. On a laissé pour ainsi dire augmenter le potentiel électrique, au lieu d'en décharger de temps en temps l'intensité par le moyen de rencontres habituelles avec les travailleurs et leurs organisations. Il est évident, alors, que quand le potentiel s'élève, la décharge devient dangereuse; elle a des répercussions violentes et menace la paix sociale et la vie de toute la collectivité.

Dans le Discours de Pie XII aux patrons, comme nous le disions, on trouve le développement logique de la doctrine sociale chrétienne. On pourrait dire encore que ce discours peut être défini comme «le message de la responsabilité sociale ». Dans Ses directives, le Souverain Pontife ne se borne pas à rappeler que, dans l'entreprise, il n'y a pas une opposition irréductible entre patrons et ouvriers, mais il souligne la communauté d'activité et d'intérêt, il suggère une communauté de responsabilités.

Dans l'entreprise, ce sont la capacité, la responsabilité et les risques qui déterminent la gradation des valeurs. Cette considération implique alors une distinction fondamentale entre les droits dont les travailleurs sont titulaires en tant que personnes humaines, indépendamment de leur capacité productive (droit au salaire familial, au logement, à l'éducation, à l'instruction professionnelle, à une stabilité de l'emploi, à la sécurité so- 
ciale) et les droits qu'on ne peut pas revendiquer du dehors, parce qu'ils sont l'expression de la capacité personnelle du travailleur (possibilité de participer plus intimement à la vie de l'entreprise sous la forme qui sera suggérée par la réalité productive).

Si ces droits pouvaient appartenir à tous, indépendamment des capacités, la thèse personnaliste serait éludée, car elle-même réclame l'épanouissement de chaque individu selon ses capacités, après qu'ont été satisfaits les droits qui sont reconnus à l'ouvrier en tant que personne humaine.

De ces considérations relatives à la valeur individuelle et sociale des responsabilités personnelles dans le monde de la production, telles qu'elles sont précisées par le document pontifical, on peut tirer les conséquences pour le règlement des autres problèmes.

L'entreprise privée paraît être le milieu le plus apte à développer le rapport de responsabilité commune, pour autant que celle-ci est «le produit vivant de la libre initiative des individus et de leurs groupes librement constitués. » Au contraire l'étatisation, fruit de l'organisation publique de l'économie, ne peut devenir la règle normale dans une société qui veut conserver la primauté à la personne humaine.

Le Bien Commun ne résulte pas de la transposition des attributions de l'individu à la collectivité, mais il demeure le produit de l'activité des individus eux-mêmes, en tant qu'elle est déployée en commun et orientée dans une même direction. C'est pour cela que le Pape Pie XII, dans le discours au Mouvement ouvrier belge, rappelle et recommande la cohésion des efforts des patrons et des ouvriers dans « un statut de droit public de la vie économique et de la vie sociale en général, selon l'organisation professionnelle ».

C'est donc la responsabilité sociale qui devient la sève de la justice sociale, là où la structure juridique de l'entreprise vient d'exclure la justice distributive. Cette responsabilité sociale, devenue orientation fondamentale dans l'organisation des forces de la production, comporte pour les patrons aussi des conséquences pratiques, surtout dès le moment qu'il y a un autre facteur dont il faut tenir compte: le temps.

\section{Les réalités économiques}

La réalité économique de l'époque (dit le Saint-Père) présente des risques à cause de la pé- nurie de capitaux et de la difficulté des échanges internationaux. Pourquoi alors, «quand il en est encore temps ne pas mettre les choses au point, dans la pleine conscience de la commune responsabilité P \ Demain, à la suite d'événements qu'on ne peut pas prévoir, la situation peut changer et rendre impossible ou bien encore plus difficile l'instauration des communes responsabilités. C'est ce qui est arrivé à propos de la suggestion faite par le Pape Pie XI, dans l'encyclique «Quadragesimo Anno », en ce qui concerne l'organisation professionnelle des différentes branches de la production.

Il parait inutile d'insister sur l'importance du fait que les mesures devront être prises en temps. Peut-être qu'ici, dans ce pays, on sent moins qu'ailleurs cette nécessité. En Europe, le communisme trouve un appui efficace à son programme dans les aspirations des masses, et, de cette façon, il peut camoufler des visées politiques précises qui ne répondent pas à une réelle volonté de progrès social. Il est nécessaire de se hâter. Aujourd'hui, disait-on dans une récente publication, il n'y a que deux solutions possibles: celle d'accepter les transferts progressifs des responsabilités aux masses syndicales révolutionnaires, qui deviendront ensuite un groupe de dirigeants durs et implacables; ou bien celle de développer des transferts progressifs et ordonnés de la responsabilité personnelle à tous ceux qui participent à la production et qui courent ainsi leur part de risques.

Cette seconde solution est sans aucun doute dans l'esprit des principes de la doctrine sociale chrétienne et correspond aux directives données par le Saint-Père aux chefs d'entreprise.

Il est encore sûr que ces directives - données en une occasion qui ont reconnu l'importance de l'organisation sociale des patrons catholiques - représentent pour eux un engagement à collaborer à la réalisation de l'ordre social chrétien, tant sur le plan de l'entreprise que sur le plan professionnel.

Le temps n'est plus où les problèmes sociaux pouvaient être laissés exclusivement aux initiatives du paternalisme patronal, bien qu'il y en ait encore quelques-uns d'attachés à cette idée dépassée. C'est pour cela que les patrons doivent voir en toute ampleur leurs devoirs économiques, productifs et sociaux, et insérer leur action quotidienne de chefs et leur effort de collaboration avec les ouvriers, dans le plan divin qui préside à l'économie de toutes les choses qui ont été créées. 